\title{
Stress distributions in bluntly-notched ceramic composite laminates
}

\author{
Varun P. Rajan*, Frank W. Zok \\ Materials Department, University of California, Santa Barbara, CA 93106, United States
}

\section{A R T I C L E I N F O}

Article history:

Received 15 August 2013

Received in revised form 26 December 2013

Accepted 19 January 2014

Available online 28 January 2014

\section{Keywords:}

A. Ceramic-matrix composites (CMCs)

B. Mechanical properties

C. Computational modeling

C. Finite element analysis

\begin{abstract}
A B S T R A C T
We present a methodology for determining stress distributions ahead of blunt notches in plates of fiberreinforced ceramic-matrix composites subject to uniaxial tensile loading, accounting for the effects of inelastic straining due to matrix cracking. The methodology is based on linear transformations of the corresponding elastic distributions. The transformations are derived from adaptations of Neuber's law for stress concentrations in inelastic materials. Comparisons are made with results computed by finite element analysis using an idealized (bilinear) form of the Genin-Hutchinson constitutive law for ceramic composite laminates. Effects of notch size and shape as well as the post-cracking tangent modulus are examined. The comparisons show that, for realistic composite properties, the analytical solutions are remarkably accurate in their prediction of stress concentrations and stress distributions, even in cases of large-scale and net-section inelasticity. Preliminary assessments also demonstrate the utility of the solution method in predicting the fields under multiaxial stressing conditions.
\end{abstract}

(c) 2014 Elsevier Ltd. All rights reserved.

\section{Introduction}

Notch sensitivity, characterized by the ratio of the notched to unnotched tensile strength, is a key performance metric of fiber-reinforced ceramic-matrix composites (CMCs). When low, it enables design of components with complex geometric features and with attendant stress concentrations using elastic analyses and low 'knock-down' factors. As in metals, stress concentrations in CMCs are mitigated by inelastic deformation, albeit through markedly different mechanisms [1,2].

The principal mechanisms operative in notched CMC laminates and their evolution with applied load are illustrated in Fig. 1. At moderate loads, matrix cracking produces inelastic strain. Its effect is to redistribute stresses at the notch tip and lower the stress concentration. At a critical load, deformation ahead of the notch becomes localized into a macroscopic band comprising a matrix crack bridged by a combination of broken and intact fibers. With additional loading, the band extends and eventually becomes unstable; that is, it grows under decreasing load. Its stability is dictated by the plate dimensions as well as its intrinsic mechanical response, characterized, for example, by a bridging traction law (Fig. 1(c)).

The present article focuses on the first of these stages. Specifically, it addresses the nature of the stress fields around notches in composite plates with $\left[0^{\circ} / 90^{\circ}\right]$ or quasi-isotropic fiber architectures subject to uniaxial tensile loading. The principal goal is to develop an analytical framework for predicting the stress fields in terms of the constituent material properties, the plate geometry

\footnotetext{
* Corresponding author. Tel.: +1 (805)893 2694.

E-mail address: varun_rajan@umail.ucsb.edu (V.P. Rajan).
}

and the applied loads. As part of a broader effort to assess notch-sensitivity, the stress fields could be used subsequently for predicting the onset of fiber bundle rupture and localization of deformation.

The article is organized in the following way. We begin with a description of the constitutive law of the composite and the key material parameters along with a description of the nature of the finite element analysis (FEA). For the purpose of generating generally applicable results and identifying important trends, the tensile stress-strain curves are idealized as being bilinear. This is followed by presentation of analytical solutions for stress concentration factors (SCF) using Neuber's law. Next, analytical solutions based on a series of proposed linear transformations to the elastic stress distributions are presented. The transformations are derived from adaptations of Neuber's law [3] for SCFs in elastic-plastic materials. The predicted SCFs and stress distributions are then assessed through comparisons with results from FEA. Effects of the postcracking tangent modulus, the degree of in-plane anisotropy, and the notch size and shape are examined. The study is directed at systems in which the matrix modulus is of the same order as the fiber modulus and which therefore exhibit minimal elastic anisotropy. This behavior is obtained in $\mathrm{SiC} / \mathrm{SiC}$ composites. Effects of elastic anisotropy, obtained in $\mathrm{C} / \mathrm{C}, \mathrm{C} / \mathrm{SiC}$ and some oxide composites $[2,4]$, are not considered.

\section{Finite element analysis}

\subsection{Constitutive law}

The material response is taken to follow a modified form of the Genin-Hutchinson constitutive model for CMC laminates [5,6]. The 
(a) Multiple matrix cracking
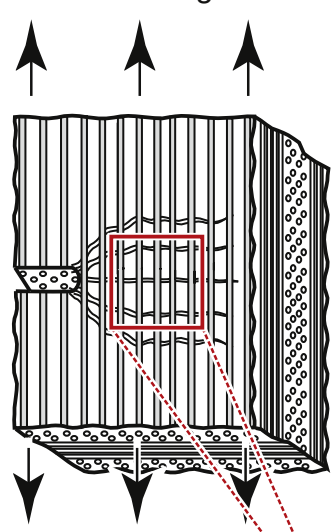

(b) Deformation localization
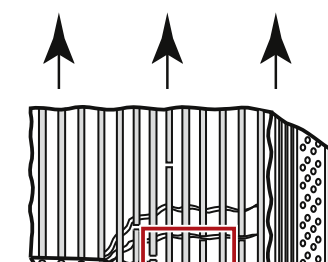

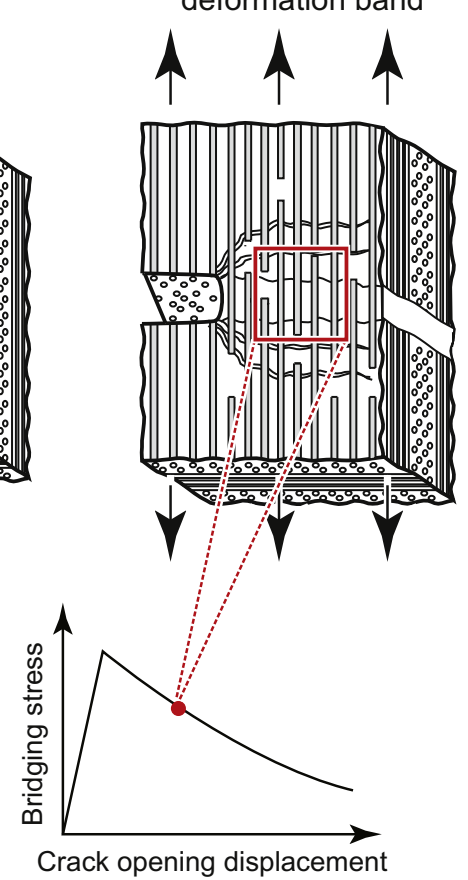

(c) Extension of deformation band

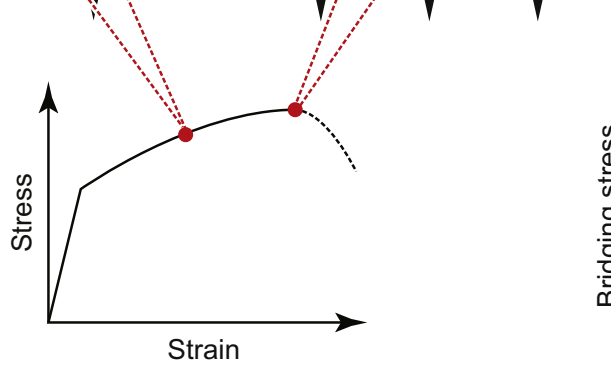

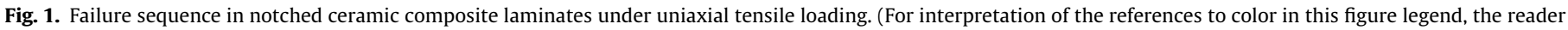
is referred to the web version of this article.)

model accounts for the macroscopic inelasticity associated with matrix damage in a phenomenological manner. The material properties are assumed to exhibit cubic in-plane symmetry, such as that obtained in balanced $\left[0^{\circ} / 90^{\circ}\right]$ laminates. The results are also applicable to the special case of quasi-isotropic laminates. For $\left[0^{\circ} / 90^{\circ}\right]$ laminates, the model is calibrated using measurements from two types of tensile tests: parallel to one of the fiber directions (in the $0^{\circ} / 90^{\circ}$ orientation) and at $45^{\circ}$ to this direction. For each test, both the longitudinal and the transverse (in-plane) strains are measured. The longitudinal and transverse strain-stress functions in the $0^{\circ} / 90^{\circ}$ orientation are denoted $f_{0}$ and $f_{0 T}$, respectively. Similarly, at $45^{\circ}$, the functions are denoted $f_{45}$ and $f_{45 T}$. Upon consideration of the special case of equibiaxial tensile loading, only three of these functions are found to be independent. In the present implementation of the model, the functions $f_{0}, f_{0 T}$, and $f_{45}$ are used for calibration ( $f_{45 T}$ being dependent on the other three). Additional details of the model are presented in A.

The subsequent numerical simulations are based on the assumption that $f_{0}$ and $f_{45}$ are bilinear functions of applied stress, each characterized by: (i) a Young's modulus, $E=E_{0}=E_{45}$, (ii) a matrix cracking stress, $\sigma_{m c}=\sigma_{m c, 0}=\sigma_{m c, 45}$, and (iii) a post-cracking tangent modulus, $E^{P}$, which may differ in the $0^{\circ}$ and $45^{\circ}$ directions (Fig. 2). The transverse strain function $f_{0 T}$ obtained in the $0^{\circ}$ $90^{\circ}$ orientation is assumed to be linear with applied stress in both the elastic and the post-cracking domains (Poisson's ratio $=0.1$ ). This assumption is supported by experimental studies which have shown that, following cracking, the transverse strain in this orientation either remains constant or decreases only very slightly in magnitude $[7,8]$. Since the Poisson's ratio of these composites is small $(<0.2)$, the elastic strains themselves are small and hence deviations from linearity have little effect on the notched composite behavior.

The baseline case is taken as one for which: (i) the normalized post-cracking tangent modulus in the $0^{\circ} / 90^{\circ}$ orientation, defined as $\alpha \equiv E_{0}^{P} / E_{0}$, is $1 / 4$ (a value representative of [ $0^{\circ} / 90^{\circ}$ ] elastically-

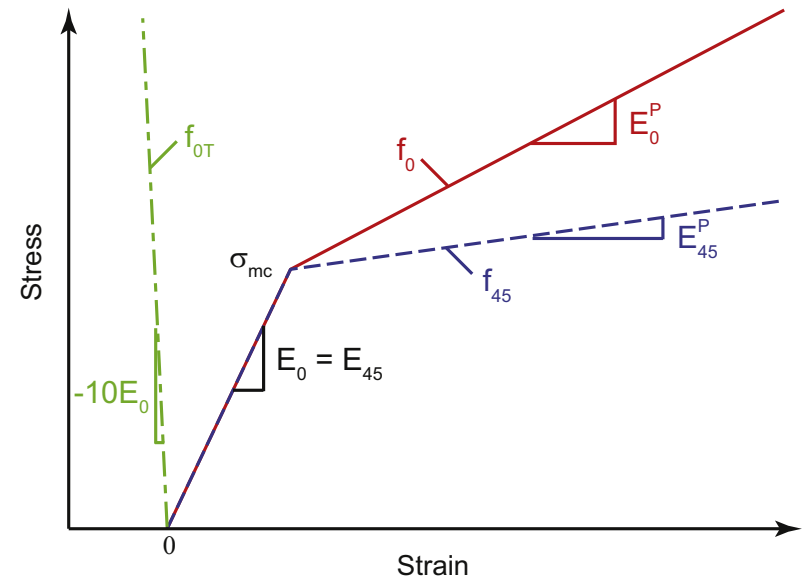

Fig. 2. Idealized stress-strain curves used for calibrating the Genin-Hutchinson constitutive model. (For interpretation of the references to color in this figure legend, the reader is referred to the web version of this article.)

isotropic laminates with a fiber volume fraction of 50\%); and (ii) the post-cracking response is isotropic $\left(\overline{E^{P}} \equiv E_{45}^{P} / E_{0}^{P}=1\right)$ and hence $f_{45}=f_{0}$. A limited parametric study is also conducted to assess the effects of both the post-cracking tangent modulus $(\alpha=1 / 10)$, and the inelastic anisotropy $\left(\overline{E^{P}}=1 / 5\right.$ and $\left.1 / 50\right)$.

When loaded in the $\pm 45^{\circ}$ direction beyond the cracking stress, the latter laminates exhibit some degree of 'scissoring': a deformation mode in which fibers rotate towards the direction of the largest principal stress. In the modified Genin-Hutchinson model, the propensity for scissoring in this orientation is characterized by a non-dimensional parameter, $0 \leqslant D_{45} \leqslant 1$ [6]. $D_{45}$ is largely controlled by the post-cracking matrix properties, which is reflected in $\overline{E^{P}}$. When the matrix provides minimal constraint against scissoring, $D_{45}$ tends towards unity; in the opposite scenario, $D_{45}$ tends 
towards zero. In the present study, we select $D_{45}=0,0.5$, and 1 for laminates with $\overline{E^{P}}=1,1 / 5$, and $1 / 50$, respectively.

\subsection{Geometry and mesh}

Finite element simulations were carried out in Abaqus Standard (Version 6-9.2, Dassault Systèmes) using the modified GeninHutchinson constitutive model as a user-material subroutine. The constitutive equations were integrated explicitly using an automatic sub-stepping algorithm discussed by Sloan et al. [9].

The specimen geometry is depicted in Fig. 3. Quarter-symmetry models with four-noded, quadrilateral, plane-stress elements were used. The plate height $H$ was set to twice the plate width (defined as $2 W$ ); this ensures that significant bending stresses do not develop in the ligament between the loaded boundary and the notch [10]. For the circular holes, the hole radius, $a$, was varied between $a / W=0.05$ and 0.5 . For elliptical holes, the principal radius, $a$, in the $x$-direction was selected to be $a / W=0.2$, and that in the $y$ direction, $b$, was set to either $b / a=1 / 3$ or 3 . Load was applied at the upper boundary in the $y$-direction. A study was performed to ensure that the stresses and strains converged with respect to mesh density.

\section{Analytical models}

\subsection{Stress concentration factors}

An analytical solution for the stress concentration factor (SCF) following the onset of inelastic straining is obtained from Neuber's law [3]. The law is based on the assumption that the stress and strain concentration factors, $k_{\sigma}$ and $k_{\epsilon}$, following the onset of local inelastic straining are related to the elastic stress concentration factor, $k_{e l}$, through

$\sqrt{k_{\sigma} k_{\epsilon}}=k_{e l}$

Neuber [3] demonstrated this relation to be strictly valid for isotropic metals subject to anti-plane shear loading. Subsequently, the law has been used extensively for predicting stress concentrations in metallic specimens for a variety of notch shapes and loading configurations [11-16].

Operationally, the law is implemented by finding the intersection point between the tensile stress-strain curve and a hyperbola described by

$\sigma \epsilon=k_{e l}^{2} \sigma_{\text {net }} \epsilon_{\text {net }}$

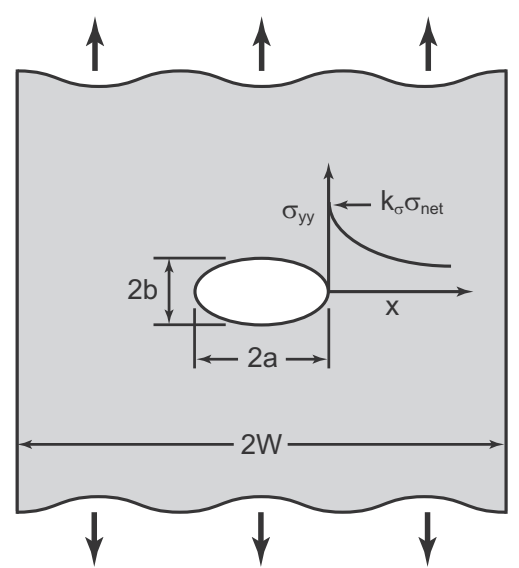

Fig. 3. Geometry of center-notched plate subjected to uniaxial tensile loading.

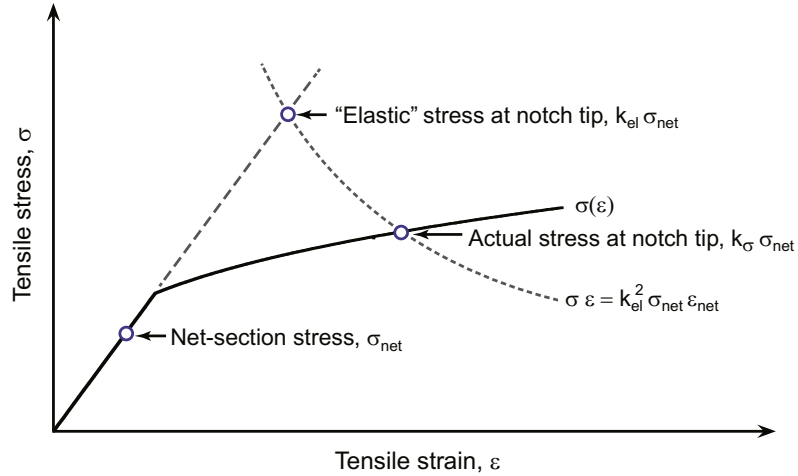

Fig. 4. Schematic illustration of the procedure used to calculate SCFs from Neuber's law. (For interpretation of the references to color in this figure legend, the reader is referred to the web version of this article.)

where $\sigma_{\text {net }}$ and $\epsilon_{\text {net }}$ are the nominal net-section values (calculated from the applied load and the uniaxial tensile stress-strain curve). The SCF is then given by the ratio of the stress at the intersection point of the two curves and the applied stress (Fig. 4). In applying the Neuber law to CMCs loaded in the $0^{\circ} / 90^{\circ}$ orientation, the relevant stress-strain curve is the one measured in that same orientation. A tacit assumption, therefore, is that the off-axis properties, as manifested in the $\pm 45^{\circ}$ tensile response, do not affect the SCF. The veracity of this assumption is addressed in due course.

For isotropic CMCs that exhibit a bilinear stress-strain response, the SCF can be evaluated analytically. Three solution regimes exist. (i) At low stresses, defined by $\beta=\sigma_{\text {net }} / \sigma_{m c} \leqslant 1 / k_{e l}$, the material remains elastic everywhere and thus $k_{\sigma}=k_{e l}$. (ii) At higher stresses, in the range $1 / k_{e l} \leqslant \beta \leqslant 1$, cracking occurs locally around the hole. Following the procedure described above, the SCF is determined by combining Eq. (2) with the tensile stress-strain curve. (That is, $\epsilon_{\text {net }}=f_{0}\left(\sigma_{\text {net }}\right)$ and $\epsilon=f_{0}(\sigma)$.) This yields (after some algebra):

$k_{\sigma}=\frac{1-\alpha+\sqrt{(1-\alpha)^{2}+4 \alpha \beta^{2} k_{e l}^{2}}}{2 \beta}$

(iii) At yet higher stresses, notably $\beta \geqslant 1$, cracking occurs across the entire section and the SCF is given by:

$k_{\sigma}=\frac{1-\alpha+\sqrt{(1-\alpha)^{2}+4 \beta(\alpha+\beta-1) k_{e l}^{2}}}{2 \beta}$

\subsection{Stress distributions}

A first approximation to the longitudinal stress distribution, $\sigma(x),{ }^{1}$ along the incipient fracture plane following the onset of inelastic straining is obtained through a straightforward modification of the elastic distribution. The modification involves application of Neuber's law to every point along this plane. This is accomplished by re-interpreting $k_{e l}$ in Eqs. (3) and (4) as the ratio of the (original) elastic stress at the point of interest, $\sigma_{e l}^{o}(x)$, to the net-section stress, $\sigma_{\text {net }}$, and $k_{\sigma}$ as the ratio of the actual stress, $\sigma(x)$, in the inelastic zone to $\sigma_{\text {net }}$. Accordingly, the modified stress along this plane in the inelastic zone is given by:

$\frac{\sigma_{\text {in }}^{o}(x)}{\sigma_{\text {net }}}=\frac{1-\alpha+\sqrt{(1-\alpha)^{2}+4 \alpha \beta^{2}\left(\sigma_{\text {el }}^{o}(x) / \sigma_{\text {net }}\right)^{2}}}{2 \beta}$

for $1 / k_{e l} \leqslant \beta \leqslant 1$, and

\footnotetext{
1 Throughout the article $\sigma(x)$ refers to the distribution in the normal stress acting in
} the direction of the applied load. 
$\frac{\sigma_{\text {in }}^{o}(x)}{\sigma_{\text {net }}}=\frac{1-\alpha+\sqrt{(1-\alpha)^{2}+4 \beta(\alpha+\beta-1)\left(\sigma_{\text {el }}^{o}(x) / \sigma_{\text {net }}\right)^{2}}}{2 \beta}$

for $\beta \geqslant 1$.

A comparison of the Neuber-modified stress distribution with that obtained from FEA for a plate with a circular hole with $a / W=0.2$ is shown in Fig. 5 . Although the proposed modification brings the elastic distribution into somewhat closer agreement with the FEA results, it underestimates both the stresses in the near-tip region and the size of the inelastic zone. The discrepancies arise because the spatial re-distribution of stress associated with inelastic straining is neglected.

The discrepancies are ameliorated through further transformations of the elastic stress distribution. The transformations build on the requirement that the net-section must support the same load before and after stress redistribution and, as a result, the size of the inelastic zone must increase in order to compensate for the reduction in stress at the notch tip. The transformations should yield stress distributions that satisfy the equilibrium condition:

$\int_{0}^{x_{i n}} \sigma_{\text {in }}(x) \mathrm{d} x+\int_{x_{\text {in }}}^{W-a} \sigma_{e l}(x) \mathrm{d} x=(W-a) \sigma_{\text {net }}$

where $\sigma_{e l}(x)$ and $\sigma_{\text {in }}(x)$ refer to the stress distributions in the elastic and inelastic zones, respectively, after transformation, and $x_{\text {in }}$ denotes the elastic-inelastic boundary. They must also satisfy the condition of continuity of stress at the inelastic zone boundary, notably

$\sigma_{\text {in }}\left(x_{\text {in }}\right)=\sigma_{\text {el }}\left(x_{\text {in }}\right)$

Numerous approaches for transforming stress distributions and estimating the size of inelastic zones have previously been proposed $[17,11,12]$ : the most well-known being Irwin's method for estimating the plastic zone size ahead of a crack in an elastic/plastic plate [18]. The transformations are typically linear, i.e. translation, scaling, or a combination thereof. To capture the stress within the elastic zone (away from the notch tip), we employ a simple translation of the original elastic stress distribution, $\sigma_{e l}^{o}(x)$, as done by Irwin [18], namely:

$\sigma_{e l}(x)=\sigma_{e l}^{o}(x+A)$

where $A$ is a positional shift along the $x$-direction, taken to be

$A=x_{\text {in }}^{o}-x_{\text {in }}$

Here $x_{i n}^{o}$ is the original estimate of the elastic-inelastic boundary, obtained by equating the elastic stress distribution to the matrix

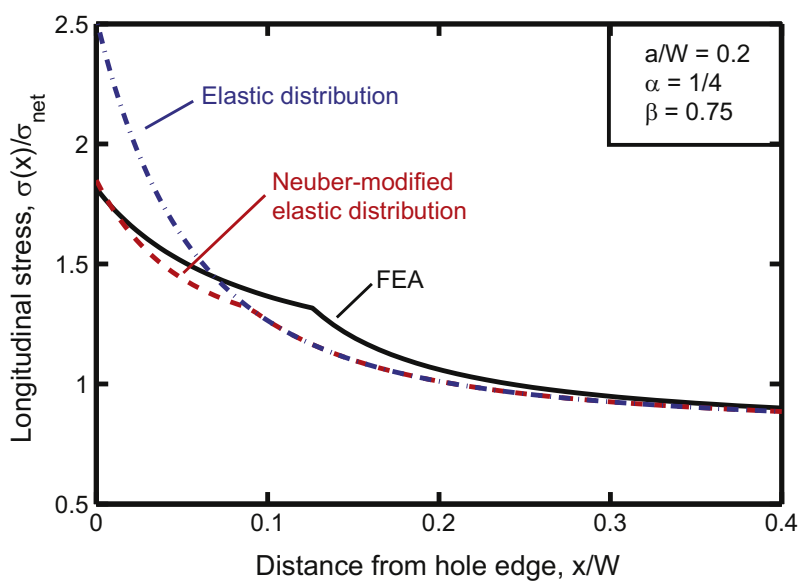

Fig. 5. Comparison of the (untransformed) Neuber-modified stress distribution with the elastic distribution and that from FEA for a plate containing a circular hole. (For interpretation of the references to color in this figure legend, the reader is referred to the web version of this article.) cracking stress (see Fig. 6), and $x_{i n}$ is obtained in the manner described below.

For the stress distribution in the inelastic zone, we start with the complete linear transformation:

$\sigma_{\text {in }}(x)=\sigma_{\text {in }}^{o}(B x+C)$

where $\sigma_{\text {in }}^{o}(x)$ is obtained from Eq. (5) or (6), as appropriate. The constants $B$ and $C$ are found by enforcing the continuity condition (Eq. (8)) in conjunction with the requirement that the stress at the notch tip (at $x=0$ ) equal that predicted by Neuber's law (Eq. (1)). Doing so yields $C=0$ and

$B=\frac{x_{i n}^{o}}{x_{i n}}$

This procedure is shown graphically in Fig. 6. The only unknown variable is the new estimate of $x_{i n}$. It can, in principle, be obtained from Eq. (7), which is nonlinear and must be solved numerically. Alternatively, in order to facilitate the development of analytical solutions, we note that, by taking

$B=\frac{k_{\sigma}}{k_{e l}}$

the resulting stress distributions (presented in Section 4.3) are in excellent agreement with those computed by FEA. Combining Eqs. (12) and (13) yields:

$x_{i n}=\frac{k_{e l}}{k_{\sigma}} x_{\text {in }}^{o}$

Combining the preceding results yields the final estimates of the stresses in the elastic and inelastic zones, notably:

$\sigma_{e l}(x)=\sigma_{e l}^{o}\left(x-x_{i n}^{o}\left[\frac{k_{e l}}{k_{\sigma}}-1\right]\right), \quad x_{i n} \leqslant x \leqslant W-a$

$\sigma_{\text {in }}(x)=\sigma_{\text {in }}^{o}\left(\frac{k_{\sigma}}{k_{e l}} x\right), \quad 0 \leqslant x \leqslant x_{\text {in }}$

where the function $\sigma_{i n}^{o}(x)$ is given by Eq. (5), $k_{\sigma}$ is from Eq. (3), and $x_{i n}$ is from Eq. (14).

Once the entire net-section has cracked, the inelastic zone size is taken to be $x_{i n}=W-a$. The equilibrium condition in this domain is

$\int_{0}^{W-a} \sigma_{i n}(x) \mathrm{d} x=(W-a) \sigma_{n e t}$

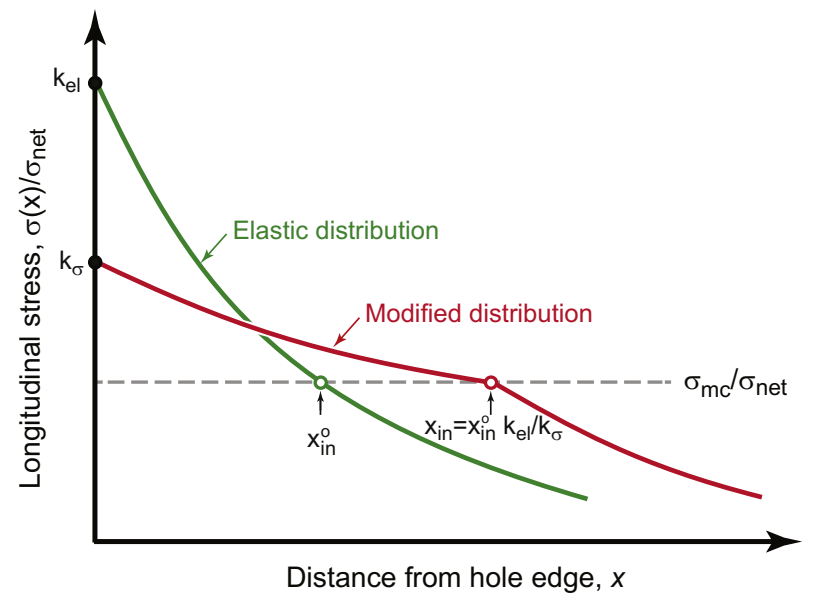

Fig. 6. Schematic representation of the transformation of the elastic distribution to account for effects of stress redistribution on both the peak stress and the size of the inelastic zone. (For interpretation of the references to color in this figure legend, the reader is referred to the web version of this article.) 
The stress distribution is again given by Eq. (15b), but with $k_{\sigma}$ and $\sigma_{i n}^{o}(x)$ now obtained from Eqs. (4) and (6), respectively.

\section{Assessment of analytical solutions}

\subsection{Preliminaries}

The accuracy of the preceding analytical solutions is evaluated by comparing the predictions with the results obtained from FEA. To this end, we use several metrics: (i) the error in the SCF, (ii) the error in $x_{i n}$, (iii) the error in satisfying the equilibrium condition (Eq. (7)), and (iv) the average error in stress along the net-section plane. In the results that follow, we employ FEA to ascertain both the inelastic and the elastic stress distributions. In principle, analytical solutions for the elastic fields could be used, thereby yielding fully-analytical solutions for the stress distributions after cracking.

Neuber's law, which forms the basis for the present model, is strictly applicable only for small-scale yielding, wherein the inelastic zone size is small compared to the notch size and the net-section width $[19,20]$. However, we find, surprisingly, that the resulting SCFs and stress distributions in bluntly-notched CMCs are in excellent agreement with the FEA results even for large-scale and net-section inelasticity. With few exceptions, this conclusion holds over the range of notch geometries considered in the present study. The basis for this conclusion follows.

\subsection{Stress concentration factors}

Comparisons of the SCFs obtained from Neuber's law (Eqs. (3) and (4)) and those from FEA for the isotropic laminate $\left(\alpha=1 / 4, \overline{E^{P}}=1\right)$ are shown in Fig. 7. Results are for circular holes with three radii $(a / W=0.05,0.2$, or 0.5$)$ and elliptical holes with two aspect ratios $(b / a=1 / 3$ or 3 , both with $a / W=0.2)$. Here the SCF is normalized by is elastic counterpart, $k_{e l}$. Matrix cracking is seen to significantly reduce the stress concentration factor at the notch tip; at the nominal net-section cracking stress $(\beta=1)$, typical reductions in stress concentration are $20-50 \%$, with larger reductions being attained for sharper notches.

The comparisons also show that Neuber's law provides a reasonably accurate description of $k_{\sigma}$ over the pertinent range of applied stress $(0 \leqslant \beta \leqslant 2)$. For all notch geometries investigated, errors in $k_{\sigma}$ are less than $10 \%$. Neuber's law performs especially well, with error less than $5 \%$, for plates with small, low aspect-ratio features. For notches that are large relative to the plate width (e.g. holes with $a / W=0.5$ ), Neuber's law somewhat over-predicts the SCF after net-section cracking, likely because of the interaction of the stress field with the plate boundaries. For sharper features, such as the elliptical hole with $b / a=1 / 3$, Neuber's law again yields slightly conservative estimates of $k_{\sigma}$ at high stress. Qualitatively similar results have been reported for notched metallic plates by Guo et al. [12], who proposed that a more accurate estimate of the SCF could be obtained by applying Neuber's law a small distance ahead of the notch-tip. However, since the errors in the present study are far lower than those reported by Guo et al. [12], this additional ad hoc adjustment is deemed unnecessary.

\subsection{Stress distribution and inelastic zones}

The proposed method for calculating the stress distribution along the net-section works exceedingly well for stress concentrating features that are small in comparison to the plate width. Typical stress distributions are shown in Fig. 8(a) for a plate with a circular hole of radius $a / W=0.2$. For an applied stress sufficient to cause large-scale cracking $(\beta=0.75)$, the method accurately
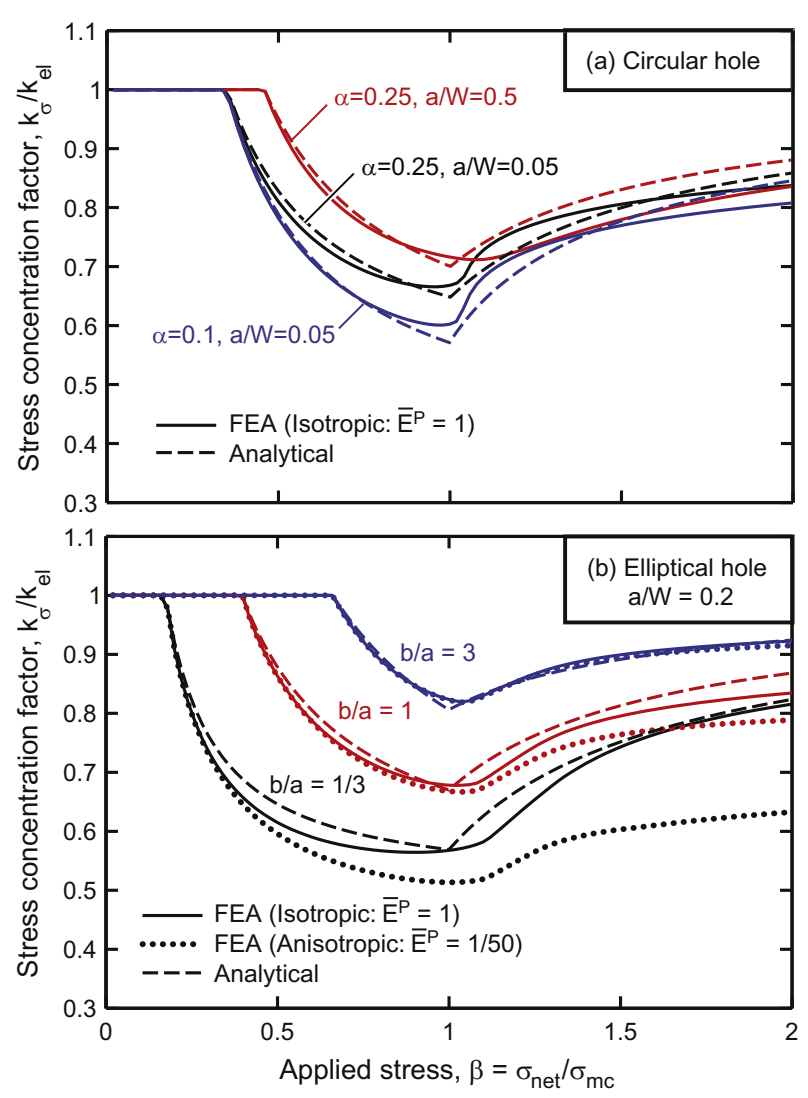

Fig. 7. Normalized stress concentration factors, $k_{\sigma} / k_{e l}$, for isotropic and anisotropic plates with (a) circular holes and (b) elliptical holes $(\alpha=1 / 4)$. (For interpretation of the references to color in this figure legend, the reader is referred to the web version of this article.)

captures both the location of the elastic-inelastic boundary and the stress distributions in the elastic and inelastic zones. Even in the domain of net-section cracking ( $\beta=1.5$ ), the stresses close to the hole are captured very well, although those near the plate edge are slightly overestimated. Clearly, the utility of the method is not limited to the restrictive case of small-scale yielding.

The accuracy of the method improves as the hole size decreases. For net-section cracking $(1 \leqslant \beta \leqslant 2)$, the normalized root-meansquared error in the predicted stresses falls in the range $1-2.5 \%$ for $a / W=0.05,3.5-7 \%$ for $a / W=0.2$, and $6.5-19 \%$ for $a / W=0.5$. To understand this result, we note that the length scale for stress decay away from the hole is governed by the hole radius, $a$. To compensate for the increase in stress near the notch (relative to the net-section stress), a reduction in stress (again, relative to the net-section stress) must occur near the plate edge. The magnitude of this stress drop is proportional to $a / W$. The present transformation method neglects this effect; consequently, stresses near the plate edge are overestimated in plates that have large stress concentrating features and that are subjected to high stresses. One important corollary is that the method should be highly accurate in the limit of infinite plates. Indeed, this conclusion is borne out by the present results.

The variations in the inelastic zone size with applied stress are plotted in Fig. 9. For moderate stress levels $(\beta \leqslant 0.9)$, excellent agreement is obtained between the model predictions and the FEA results. In this domain, the error between the two is less than $5 \%$. Similar agreement is obtained for the other notch geometries considered in this study (not shown); the one exception is the plate with an elliptical hole with $b / a=3$, for which the error is still less than $10 \%$. Once the net-section cracking condition is met $(\beta=1)$, 

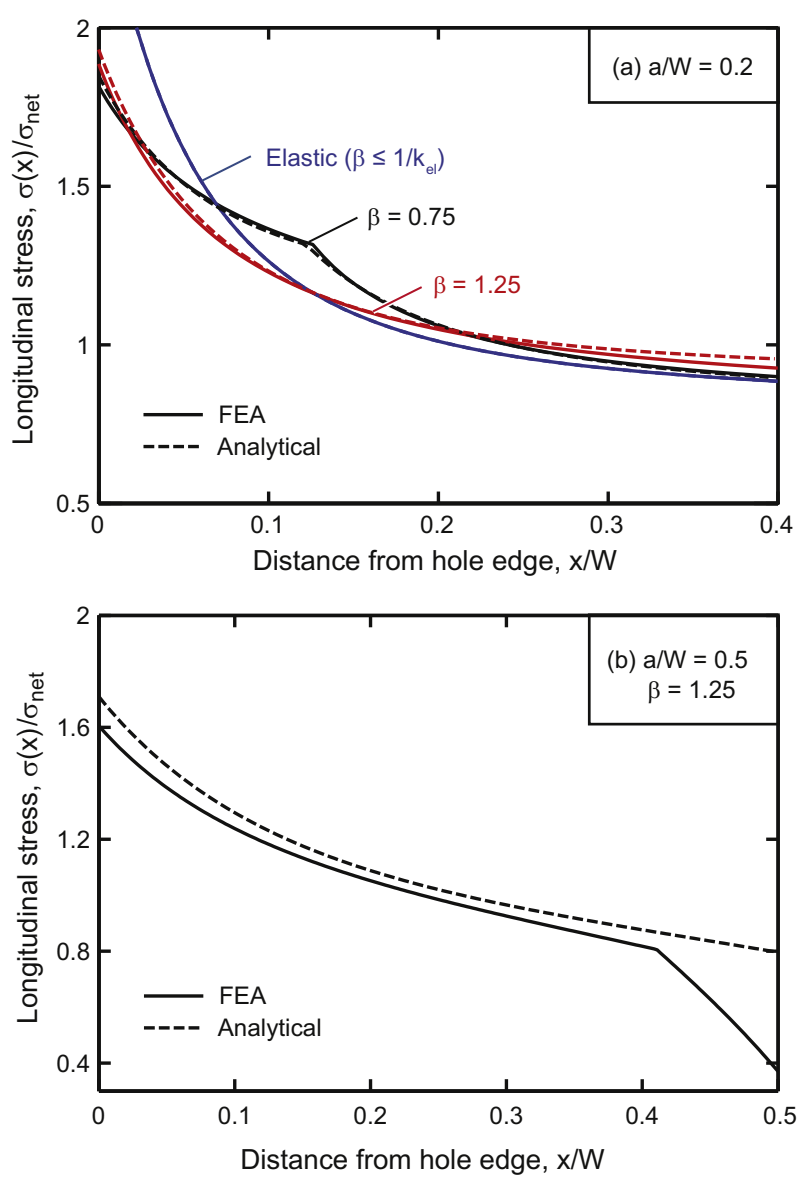

Fig. 8. Stress distributions in isotropic plates $\left(\alpha=1 / 4, \overline{E^{P}}=1\right)$ with circular holes of radius: (a) $a / W=0.2$ and (b) $a / W=0.5$. Results are shown for stress levels representative of both large-scale and net-section inelasticity. (For interpretation of the references to color in this figure legend, the reader is referred to the web version of this article.)

the analytical model predicts that the inelastic zone encompasses the entire net-section $\left(x_{i n}=W-a\right)$. In reality, because of the stress reduction effect that occurs for larger holes, this boundary may exist within the bounds of the plate even when $\beta=1$. Thus the computed transition at and slightly beyond $\beta=1$ is somewhat more gradual, especially for large $a / W$.

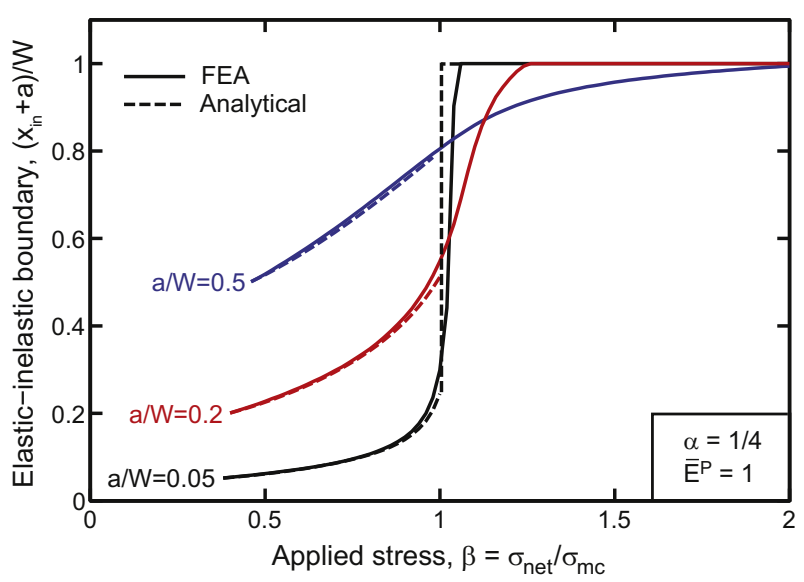

Fig. 9. Variation in the elastic-inelastic boundary with applied stress for isotropic plates containing circular holes. (For interpretation of the references to color in this figure legend, the reader is referred to the web version of this article.)
The models for the stress distribution and the inelastic zone size perform equally well for plates with elliptical holes (Fig. 10). Here, again, the near-tip stresses are captured very well by the analytical model; the errors are slightly greater near the plate edge at high stress $(\beta \geqslant 1)$.

Because stresses at the plate edge tend to be overestimated by the present model, the equilibrium condition (Eqs. (7) and (16)) is only approximately satisfied. For plates with larger notches, the net-section reaction force predicted by the analytical model is approximately 2-6\% larger than the applied load at high applied stresses. Enforcing the equilibrium condition, by numerically solving Eq. (7) to find $x_{i n}$, brings the stresses near the plate edge into somewhat better agreement with the FEA results. However, this improvement is offset by poorer agreement in the stress near the notch tip, particularly for sharp notches. Since the near-tip stresses are arguably most important in failure prediction, this alternative approach is not recommended.

\subsection{Effects of post-cracking tangent moduli}

Neuber's law assumes isotropic mechanical response. When applied to anisotropic laminates, it tacitly neglects the off-axis properties (at $45^{\circ}$ ). This assumption is assessed by examining the effects of the post-cracking tangent moduli, $E_{0}^{P}$ and $E_{45}^{P}$, on the correspondence between the predicted and computed stress fields and, in turn, on the accuracy of the proposed transformation method. Finite element results for the SCF for $\alpha=1 / 10$ (shown in Fig. 7(a)) reveal only slight increases in error relative to those obtained for the higher hardening laminate $(\alpha=1 / 4)$. Analogous

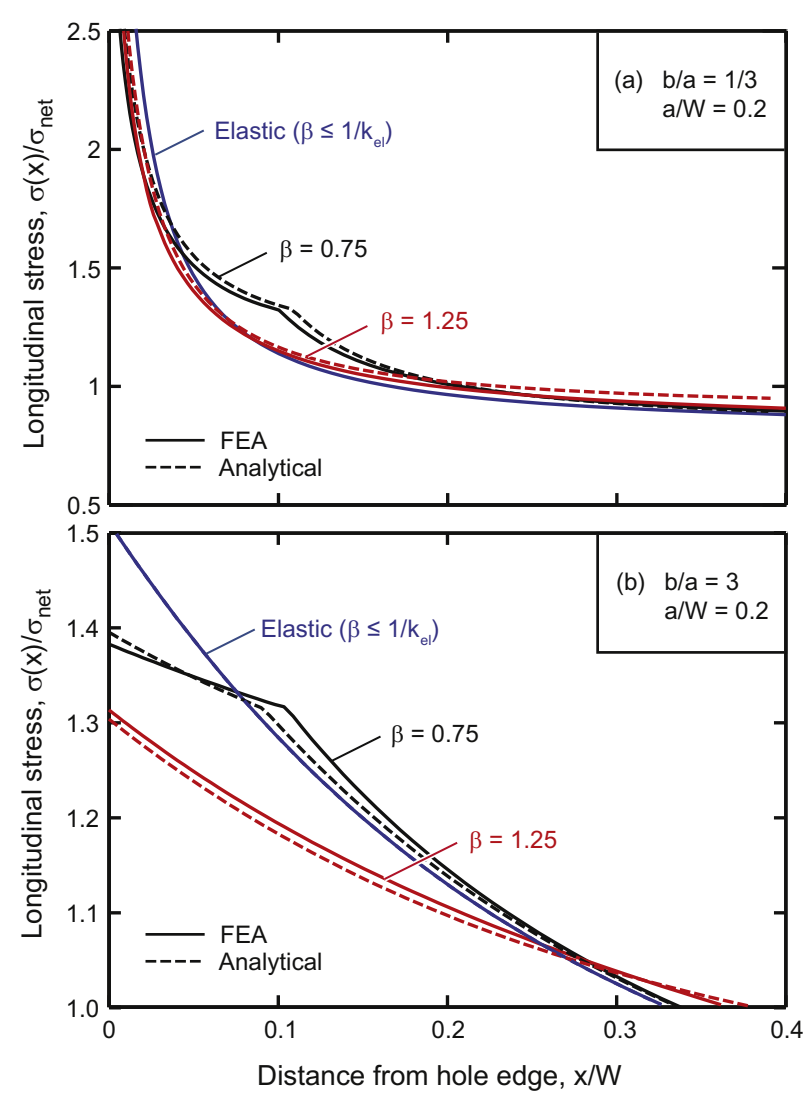

Fig. 10. Stress distributions in isotropic plates $\left(\alpha=1 / 4, \overline{E^{P}}=1\right)$ with elliptical holes of aspect ratio: (a) $b / a=1 / 3$ and (b) $b / a=3$. Results are shown for stress levels representative of both large-scale and net-section inelasticity. (For interpretation of the references to color in this figure legend, the reader is referred to the web version of this article.) 
results for the SCFs in a highly anisotropic laminate $\left(\overline{E^{P}}=1 / 50\right)$ are plotted on Fig. 7(b) and the stress distribution at one stress level $(\beta=2)$ is plotted in Fig. 12. Comparisons with the analytical results reveal that, for features with inherently low SCF (say, $b / a \geqslant 1$ ), the errors in the predicted SCFs are no greater than those for the isotropic laminate. For features that yield higher stress concentrations (e.g. elliptical hole with $b / a=1 / 3$ ), the errors in SCF are somewhat greater than those of the isotropic laminate, although the predicted SCF values are conservative over the entire stress range (i.e. lower than those obtained from FEA).

The origin of the differences in behavior of the isotropic and anisotropic laminates at high stress can be elucidated from examination of strain distributions obtained from FEA. Two sets of results, for $\overline{E^{P}}=1$ and $1 / 50$, are shown in Fig. 11 . The comparisons show that, at high stresses (well beyond the onset of cracking), the shear strain $\gamma_{x y}$ in the notch-tip region adjacent to the net-section plane is almost twice that obtained at the same location in the isotropic laminate (see, for example, the points indicated on Fig. 11(c) and (d)). The additional shear strain has the effect of mitigating the axial tensile strains directly ahead of the notch tip. Moreover, the somewhat elongated shape of the zone of high shear is reminiscent of the deformation preceding the formation of a shear band. Indeed, the increased propensity for shear banding in the anisotropic laminate gives rise to the reduced SCF. Analogous effects have been found in some anisotropic fiber-reinforced polymer-matrix composites [21] and ceramic/metal laminates [22]. Examinations of other strain distributions (not shown) reveal that the notch shape also plays a role: sharp notches inducing larger shear strains than circular holes.

\subsection{Extension to multiaxial loading}

Here we present a preliminary assessment of the utility of the analytical method in predicting SCFs and stress distributions under multiaxial loading conditions. It is motivated in part by reports that, in metallic alloys, Neuber's law yields inaccurate results when

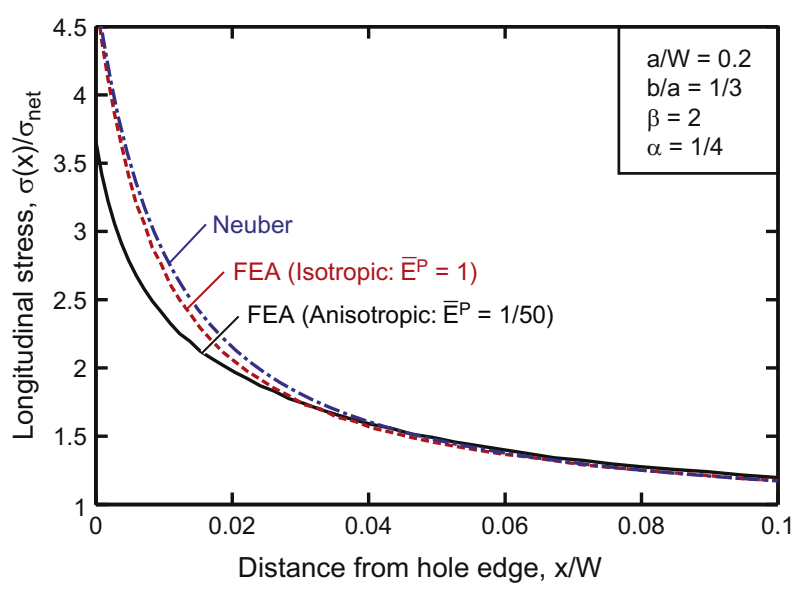

Fig. 12. Near-tip stresses under net-section inelasticity in both isotropic and anisotropic laminates. (For interpretation of the references to color in this figure legend, the reader is referred to the web version of this article.)

stress multiaxiality is significant $[12,15,23,17,24,14]$. The errors are attributed to effects of stress multiaxiality on plastic straining in metals [15]. Local stress multiaxiality can arise in plates that are sufficiently thick to create plane strain conditions at the notch tip or when the applied loads themselves are multiaxial. Our expectation at the outset is that such effects should be less important for isotropic ceramic composite laminates. This expectation is based on three observations. First, inelasticity (i.e. cracking) in ceramic composites is driven by the maximum principal tension (in contrast, metal plasticity is driven by the deviatoric stress). Second, the coupling of axial stresses with transverse strains is weak in ceramic composites, both in the elastic and the inelastic domains [5]. Third, plane strain conditions are not obtained in CMC plates. Thus we expect the analytical method to remain applicable when uniaxial tension is combined with other in-plane loadings, provided that the loading remains proportional.

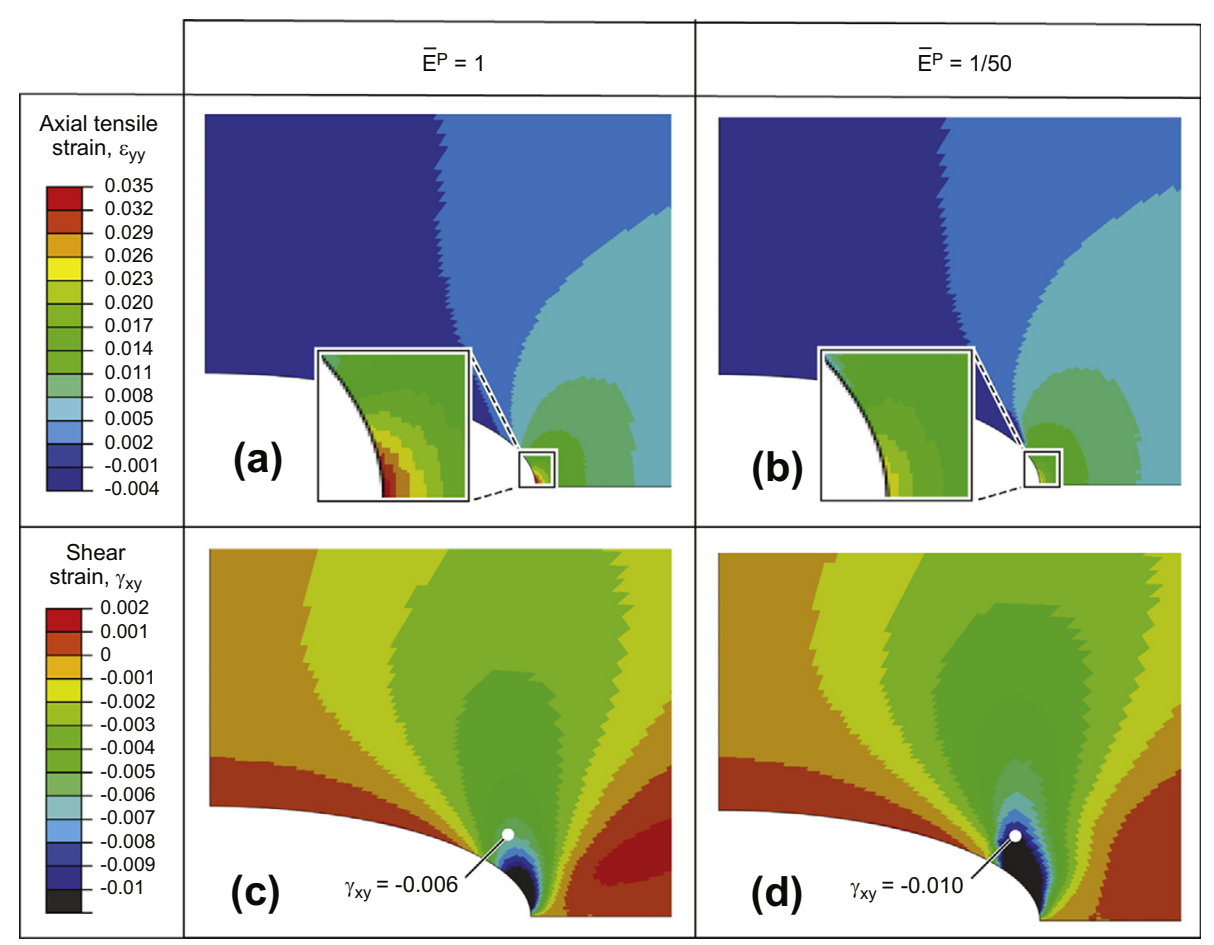

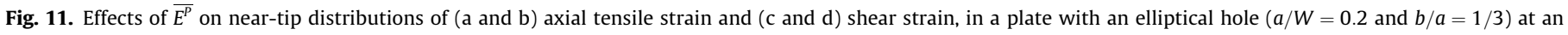
applied stress of $\beta=2(\alpha=1 / 4)$. (For interpretation of the references to color in this figure legend, the reader is referred to the web version of this article.) 

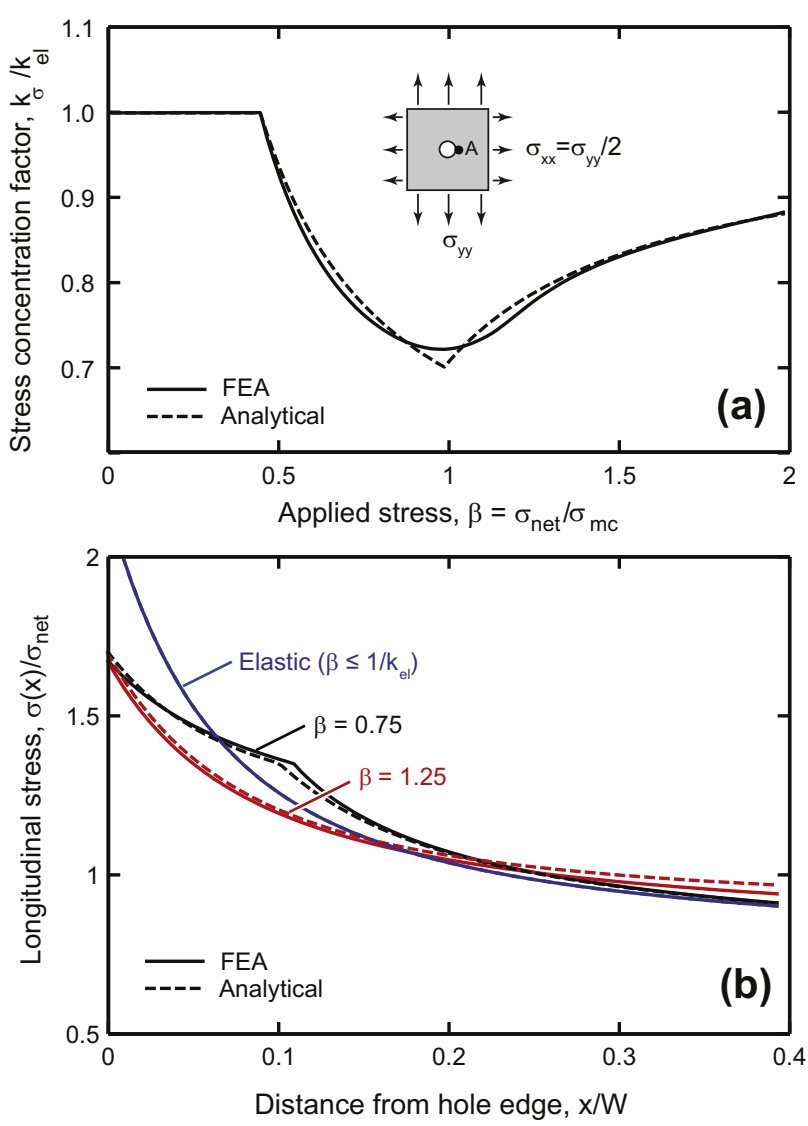

Fig. 13. Effects of stress multiaxiality on (a) the SCF (at point A in the inset) and (b) the stress distribution in an isotropic plate with circular hole $(a / W=0.2, \alpha=1 / 4)$. (For interpretation of the references to color in this figure legend, the reader is referred to the web version of this article.)

To assess this hypothesis, we consider the case of proportional biaxial loading of a square plate $(W=H)$ with a circular center hole $(a / W=0.2)$. The applied stress in the $x$-direction (at $\left.0^{\circ}\right)$ is half of that in the $y$-direction. The FEA is otherwise identical to that described previously. The variation in SCF with applied stress is plotted in Fig. 13(a) and representative stress distributions are shown in Fig. 13(b). The correlations between the analytical predictions and the FEA results are very good: comparable to those obtained for uniaxial tensile loading. We conclude that, for isotropic CMC laminates, the current analytical method should find utility in predicting stress concentrations and stress distributions in scenarios involving multiaxial in-plane loading.

\section{Conclusions}

A methodology for determining stresses ahead of blunt notches in plates of CMC laminates has been developed. The methodology is based on a series of transformations of the corresponding elastic distribution. Briefly, within the inelastic zone, both the (original) elastic stress and the position coordinate are scaled by $k_{\sigma} / k_{e l}$ : the latter obtained directly from Neuber's law. Within the elastic zone, the position coordinate of the original elastic stress is simply shifted by $x_{i n}^{o}\left(k_{e l} / k_{\sigma}-1\right)$. The resulting solutions are in remarkably good agreement with the FEA results for isotropic laminates $\left(E^{P}=1\right)$ with realistic post-cracking tangent moduli $(\alpha \geqslant 1 / 10)$, especially for small, low aspect ratio notches $(b / a \sim 1)$. Surprisingly, the agreement is largely maintained even when the entire net section becomes inelastic $(\beta>1)$. The predicted stresses for highly anisotropic laminates $\left(E^{P}=1 / 50\right)$ are similarly accurate for low aspect ratio notches and moderate applied stress levels; for sharp notches and high stresses, the predictions overestimate the near-tip stresses. The discrepancies are attributable to the larger shear strains obtained at the notch tip in the latter scenarios: an effect not captured by the model. Finally, preliminary assessments indicate that the present methodology should find utility in predicting the notch-tip fields in isotropic laminates under multiaxial stressing conditions.

\section{Acknowledgements}

This work was supported by the Pratt \& Whitney Center of Excellence at the University of California, Santa Barbara (monitored by Douglas Berczik). VPR was supported in part by a National Defense Science and Engineering Graduate Fellowship.

\section{Appendix A. Modified Genin-Hutchinson constitutive law for CMC laminates}

The constitutive model $[5,6]$ requires as inputs two sets of functions, which relate stress and strain along axes of material symmetry. One set $\left(f_{0}, f_{0 T}\right)$, represents the stress-strain measurements in a $0^{\circ}$ tension test, with $f_{0}$ obtained from the longitudinal strains (parallel to the loading direction) and $f_{0 T}$ obtained from the transverse strains. Similarly, the other set $\left(f_{45}, f_{45 T}\right)$ is obtained from a tension test conducted at $\pm 45^{\circ}$ to the two fiber directions.

In the elastic regime, the stress state can be computed from the strain state using the stiffness matrix for elastic laminates in plane stress. This matrix requires the elastic constants $E_{0}, E_{45}, v_{0}$, and $v_{45}$ of the laminate.

For laminates where the matrix cracking stresses and strains are identical in the $0^{\circ}$ and $45^{\circ}$ directions, matrix cracking is assumed to occur when the first principal stress exceeds the matrix cracking stress. In material elements that have undergone cracking, the model relates increments of stress to increments of strain via a tangent stiffness matrix that depends on the current stress state. The current strain vector, $\left[\epsilon_{x x}, \epsilon_{y y}, \gamma_{x y}\right]$, is rotated into the principal axes, which yields

$\boldsymbol{\epsilon}=\left[\epsilon_{I}, \epsilon_{I I}, 0\right]$

where the principal axes of strain are denoted $I$ and II. The angle of the principal strain axes with respect to the fiber axes is denoted $\theta_{\epsilon}$.

The current stress vector, $\left[\sigma_{x x}, \sigma_{y y}, \sigma_{x y}\right]$, is also rotated into the principal strain axes:

$\boldsymbol{\sigma}=\left[\sigma_{I}, \sigma_{I I}, \tau\right]$

where $\tau$ may be nonzero if the laminate is anisotropic, and thus the principal stress and strain axes are not aligned.

Finally, the increments in strain, $\left[\mathrm{d} \epsilon_{x x}, \mathrm{~d} \epsilon_{y y}, \mathrm{~d} \gamma_{x y}\right]$, and stress, $\left[\mathrm{d} \sigma_{x x}, \mathrm{~d} \sigma_{y y}, \mathrm{~d} \tau_{x y}\right]$, are also rotated into $\theta_{\epsilon}$ :

$\mathbf{d} \epsilon=\left[\mathrm{d} \epsilon_{I}, \mathrm{~d} \epsilon_{I I}, \mathrm{~d} \gamma\right]$

$\mathbf{d} \boldsymbol{\sigma}=\left[\mathrm{d} \sigma_{I}, \mathrm{~d} \sigma_{I I}, \mathrm{~d} \tau\right]$

where $\mathrm{d} \gamma$ is small compared to $\mathrm{d} \epsilon_{I, I I}$ since the straining is nearly proportional.

Shear-extension coupling is assumed to be negligible, so that the tangent stiffness matrix, which relates $\mathbf{d} \boldsymbol{\sigma}$ and $\mathbf{d} \boldsymbol{\epsilon}$, is orthotropic. The increment in shear stress is related to the increment in shear strain through

$\mathrm{d} \tau=\frac{\sigma_{I}-\sigma_{I I}}{2\left(\epsilon_{I}-\epsilon_{I I}\right)} \mathrm{d} \gamma$

The stiffness matrix that relates the principal stress increment to the principal strain increment is obtained by interpolating between the stiffness matrices for $0^{\circ}$ and $45^{\circ}$ : 


$$
\left[\begin{array}{l}
\mathrm{d} \sigma_{I} \\
\mathrm{~d} \sigma_{I I}
\end{array}\right]=\left(\left[\boldsymbol{S}^{\mathbf{0}}(\boldsymbol{\sigma})\right]^{-1} \cos ^{2}\left(2 \theta_{\epsilon}\right)+\left[\boldsymbol{S}^{45}(\boldsymbol{\sigma})\right]^{-1} \sin ^{2}\left(2 \theta_{\epsilon}\right)\right)\left[\begin{array}{l}
\mathrm{d} \epsilon_{I} \\
\mathrm{~d} \epsilon_{I I}
\end{array}\right]
$$

where the compliance matrices, $\boldsymbol{S}$, at $0^{\circ}$ and $45^{\circ}$ are given by

$$
\boldsymbol{S}^{\theta}=D_{\theta}\left[\begin{array}{cc}
f_{\theta}^{\prime}\left(\sigma_{I}\right) & f_{\theta T}^{\prime}\left(\sigma_{I}\right) \\
f_{\theta T}^{\prime}\left(\sigma_{I}\right) & f_{\theta}^{\prime}\left(\sigma_{I}\right)
\end{array}\right]+\left(1-D_{\theta}\right)\left[\begin{array}{cc}
f_{\theta}^{\prime}\left(\sigma_{I}\right) & f_{\theta T}^{\prime}\left(\sigma_{I I}\right) \\
f_{\theta T}^{\prime}\left(\sigma_{I}\right) & f_{\theta}^{\prime}\left(\sigma_{I I}\right)
\end{array}\right]
$$

where the primes denote differentiation with respect to stress. The parameter $D_{\theta}$ characterizes the tendency of the laminate to 'scissor' when the principal strains are oriented at $\theta[6]$. In the $0^{\circ}$ direction, the fibers are aligned with the load axis, and thus $D_{0}=0$. In contrast, in the $45^{\circ}$ direction, the laminate may undergo scissoring. For a quasi-isotropic laminate (e.g. $\left[0^{\circ} / \pm 45^{\circ} / 90^{\circ}\right]$ ), $D_{45}$ must equal 0 , since the $0^{\circ}$ and $45^{\circ}$ directions are equivalent. In contrast, for a cross-ply $\left(\left[0^{\circ} / 90^{\circ}\right]\right)$ laminate, deformation is expected to be dominated by the scissoring mechanism; therefore, $D_{45}$ should be close to unity. (The precise value is unimportant, because stresses that arise from on-axis loading of a notched plate are insensitive to $\left.D_{45}[6].\right)$

Upon consideration of equibiaxial tensile loading $\left(\sigma_{I}=\sigma_{I I}=\sigma\right)$, an equation relating the functions $f$ is obtained:

$f_{0}(\sigma)+f_{0 T}(\sigma)=f_{45}(\sigma)+f_{45 T}(\sigma)$

Hence, only three of the stress-strain curves are independent functions.

\section{References}

[1] Evans AG, Zok FW. The physics and mechanics of fibre-reinforced brittle matrix composites. J Mater Sci 1994;29(15):3857-96.

[2] Cady C, Heredia FE, Evans AG. In-plane mechanical properties of several ceramic-matrix composites. J Am Ceram Soc 1995;78(8):2065-78.

[3] Neuber $\mathrm{H}$. Theory of stress concentration for shear-strained prismatical bodies with arbitrary nonlinear stress-strain law. J Appl Mech 1961;28(4):544-50.

[4] Heathcote JA, Gong XY, Yang JY, Ramamurty U, Zok FW. In-plane mechanical properties of an all-oxide ceramic composite. I Am Ceram Soc 1999;82(10):2721-30.
[5] Genin GM, Hutchinson JW. Composite laminates in plane stress: constitutive modeling and stress redistribution due to matrix cracking. J Am Ceram Soc 1997;80(5):1245-55.

[6] Rajan VP, Zok FW. Remediation of a constitutive model for ceramic composite laminates. Composites Part A 2013;52:80-8.

[7] Lynch CS, Evans AG. Effects of off-axis loading on the tensile behavior of a ceramic-matrix composite. J Am Ceram Soc 1996;79(8):3113-23.

[8] McNulty JC, Zok FW, Genin GM, Evans AG. Notch-sensitivity of fiber-reinforced ceramic-matrix composites: effects of inelastic straining and volumedependent strength. J Am Ceram Soc 1999;82(5):1217-28.

[9] Sloan SW, Abbo AJ, Sheng D. Refined explicit integration of elastoplastic models with automatic error control. Eng Comput 2001;18(1/2):121-54.

[10] Genin GM, Hutchinson JW. Failure at attachment holes in brittle matrix laminates. J Compos Mater 1999;33(17):1600-19.

[11] Agnihotri G. Calculation of elastic-plastic strains and stresses in notches under torsion load. Eng Fract Mech 1995;51(5):823-35.

[12] Guo W, Wang CH, Rose LRF. Elastoplastic analysis of notch-tip fields in strain hardening materials. Melbourne, Victoria: Report DSTO-RR-0137, DSTO Aeronautical and Maritime Research Laboratory; 1998.

[13] Jiang Y, Xu B. Deformation analysis of notched components and assessment of approximate methods. Fatigue Fract Eng Mater Struct 2001;24(11):729-40.

[14] Moftakhar A, Buczynski A, Glinka G. Calculation of elasto-plastic strains and stresses in notches under multiaxial loading. Int J Fract 1995;70(4):357-73.

[15] Zeng Z, Fatemi A. Elasto-plastic stress and strain behaviour at notch roots under monotonic and cyclic loadings. J Strain Anal Eng Des 2001;36(3):287-300.

[16] Härkegard G, Mann T. Neuber prediction of elastic-plastic strain concentration in notched tensile specimens under large-scale yielding. J Strain Anal Eng Des 2003;38(1):79-94.

[17] Glinka G. Calculation of inelastic notch-tip strain-stress histories under cyclic loading. Eng Fract Mech 1985;22(5):839-54.

[18] Irwin GR. Plastic zone near a crack and fracture toughness. In: Proceedings of the seventh sagamore conference; 1960. p. IV-63.

[19] Rice JR. Mechanics of crack tip deformation and extension by fatigue. Philadelphia, PA: American Society for Testing and Materials; 1967. p. 247-311.

[20] Zappalorto M, Lazzarin P. Analytical study of the elastic-plastic stress fields ahead of parabolic notches under antiplane shear loading. Int J Fract 2007; 148(2):139-54.

[21] Yang O Cox B. Cohesive models for damage evolution in laminated composites. Int J Fract 2005;133(2):107-37.

[22] Chan KS, He MY, Hutchinson JW. Cracking and stress redistribution in ceramic layered composites. Mater Sci Eng A 1993;167(1/2):57-64.

[23] Molski K, Glinka G. A method of elastic-plastic stress and strain calculation at a notch root. Mater Sci Eng 1981;50(1):93-100.

[24] Hoffman M, Seeger T. A generalised method for estimating multiaxial elasticplastic notch stresses and strains. Part I: Theory. J Eng Mater Technol 1985:107(4):250-4. 